\title{
QSAR studies of some anilinoquinolines for their antitumor activity as EGFR inhibitors
}

\author{
Shweta Sharma ${ }^{1}$, Vijay K. Agrawal ${ }^{2}$, Basheerulla Shaik ${ }^{2}$, and Anita K. ${ }^{3}$ \\ ${ }^{1,3}$ Department of Chemistry, Career College, Bhopal-462002, Madhya Pradesh, India \\ ${ }^{2}$ National Institute of Technical Teachers' Training \& Research, Bhopal
}

\begin{abstract}
Quantitative Structure-Activity Relationship studies has been performed on some anilinoquinolines . A variety of parameters including $2 D$ - autocorelation, RDF, 3D- MoRSE, WHIM and GETAWAY parameters have been chosen for modeling the antitumor activity of these compounds. The multiple regression analysis reveals that the seven -parametric model is the best for modeling the activity of the compounds under present study. This model has been tested by using cross validated parameters. The results are also discussed on the basis of ridge regression.
\end{abstract}

Keywords: QSAR, NCSS, EGFR, Ridge regression.

\section{Introduction}

Cancer is now a day's used as a generic term describing a group of about 120 different diseases, which can affect any part of the body and defined as the state characterized by the uncontrolled growth and invasion of normal tissues and spread of cells [1]. According to WHO reports cancer is a leading cause of premature death worldwide, accounting for 7.6 million deaths (around 13\% of all deaths) only in 2008. The deaths from cancer worldwide are projected to continue rising, reaching an estimated 13.1 million in 2030 (WHO 2012) [2]. A number of natural and synthetic products have been found to exhibit anticancer activity against tumor cell lines [3-7].

It is worth mentioning that epidermal growth factor receptor (EGFR) is a rational target for antitumor strategies. The EGFR is expressed or highly expressed in a variety of human tumors including non-small cell lung cancer (NSCLC), breast, head and neck, gastric, colorectal, esophageal, prostate, bladder, renal, pancreatic, and ovarian cancers [8]. Particularly, a large number of compounds have been synthesized and evaluated as EGFR inhibitors, with special attention being paid to compounds having a phenyl amino pyrimidine moiety in their structures [9-13]. Evidently Anilinoquinolines are well known EGFR inhibitors as demonstrated in various studies [14-16]. In silico QSAR studies were performed by Pasha et al., [17] on a series of EGFR inhibitory anilinoquinolines . In the study they have reported HQSAR, DFT-based QSAR, ligand - based 3D- QSAR, and receptor- guided HQSAR analysis of 58 anilinoquinoline derivatives using various parameters. In the present study we are also using the same data set of compounds with $\mathrm{pIC}_{50}$ activities, but selecting different independent variables.

\section{Computational Details}

The structural details of the 58 anilinoquinolines derivatives with EGFR inhibitory activity $\mathrm{pIC}_{50}$ have been reported in table 1. The calculated parameters have been summarized in table 2. From the large pool of 2D-autocorrelation parameters, RDF, 3D MoRSE, WHIM and GETAWAY descriptors we have selected a few to carryout multiple regression analysis using variable selection method. For calculating the parameters we have used Dragon software [18] .From all the calculated descriptors, useful descriptors were generated by variable selection of descriptors in multiple regression analysis using NCSS software [19]. The intercorrelatedness among the descriptors and their correlation with the activity values $\mathrm{pIC}_{50}$ is presented in Table 3 . All the statistically significant models along with their quality have been summarized in table 4 . This table also includes the values of Poglianis quality factor Q [20-22], which is the ratio of $\mathrm{R}$ and $\mathrm{Se}(\mathrm{Q}=\mathrm{R} / \mathrm{Se})$.

\section{Results and Discussion}

The regression analysis of the data set yielded several statistically significant models. However, on the basis of highest $\mathrm{R}^{2}$ value, we propose following statistically significant models to be best for modeling the EGFR inhibitory activity:

Five variable model (Model no.5, Table no.4)

The Five -parametric model having maximum $\mathrm{R}^{2}$ value 0.7779 is as below:

$\mathrm{pIC}_{50}=-22.2852( \pm 3.3394) \mathrm{G} 3 \mathrm{~m}-7.6033( \pm 0.8275)$ MATS1e $+8.2819( \pm 1.6698)$ MATS3p +

0.0356( \pm 0.0101$)$ RDF095s- $0.4418( \pm 0.1033)$ RDF110u +12.9503

$\mathrm{N}=58, \mathrm{R}^{2}=0.7779, \quad \mathrm{R}_{\mathrm{A}}{ }_{\mathrm{A}}=0.7566, \mathrm{Se}=0.0930, \mathrm{~F}=36.429, \mathrm{Q}=9.484$ 
Six variable model (Model no.6, Table no.4)

When $\mathrm{H} 1 \mathrm{i}$ is added to the above model a six-parametric model with $\mathrm{R}^{2}=0.8177$ is obtained as below: $\mathrm{pIC}_{50}=-18.9191( \pm 3.2170) \mathrm{G} 3 \mathrm{~m}+1.6503( \pm 0.7941) \mathrm{H} 1 \mathrm{i}-7.4943( \pm 0.7577) \mathrm{MATS} 1 \mathrm{e}+9.8815( \pm 1.6009)$ MATS3p+ 0.0477( \pm 0.0099$) R D F 095 s-0.4949( \pm 0.0958)$ RDF110u +5.4334 $\mathrm{N}=58, \mathrm{R}^{2}=0.8177, \mathrm{R}_{\mathrm{A}}=0.7963, \mathrm{Se}=0.0851, \mathrm{~F}=38.134, \mathrm{Q}=10.626$

Seven variable model (Model no.7, Table no.4)

When Mor29u combined with the above six-parametric model a seven- parametric model is yielded with improved $\mathrm{R}^{2}$ value.

$\mathrm{pIC}_{50}=-20.5758( \pm 2.8191) \mathrm{G} 3 \mathrm{~m}+3.2238( \pm 0.7024) \mathrm{H} 1 \mathrm{i}-7.4577( \pm 0.6576)$ MATS1e $+11.0537( \pm 1.4168)$ MATS3p-2.1918( \pm 0.5205$)$ Mor29u+0.0607( \pm 0.0091$) R D F 095 s-0.7058( \pm 0.0971)$ RDF110u +4.8803

(3)

$\mathrm{N}=58, \mathrm{R}^{2}=0.8654, \quad \mathrm{R}_{\mathrm{A}}^{2}=0.8466, \mathrm{Se}=0.0738, \mathrm{~F}=45.943, \mathrm{Q}=12.605$

It is evident that the value of $\mathrm{R}^{2}$ is drastically increases from six-parametric model to the seven-parametric model. This means that seven-variable model is statistically better than previous one. Consequently, we observed that the seven-parametric model is the best for modeling the activity.

Further confirmation is obtained by estimating the $\mathrm{pIC}_{50}$ activity using model- 7 which is reported in Table 5 . The estimated values are in good agreement with observed values. When observed activity values are plotted against estimated values, we obtained a graph which is reported in Fig. 2. The predictive power of the model comes out to be 0.8654 . To validate the obtained model cross validation has been done by LOO method, and obtained values are reported in table 6 . The highest $\mathrm{R}^{2} \mathrm{CV}$ for the model 7 shows that this is the most appropriate model for modeling $\mathrm{pIC}_{50}$ value of compounds used in the present study. For any kind of possible defect we have calculated variance inflation factor, tolerance and condition number for various parameters using VIF plot which is given in Table 7. All the parameters show the value within the permissible limit. Therefore, the model is free from any kind of defect. Ridge trace suggests that there is no co- linearity in the model.

\section{Conclusion}

1. 2D -autocorrelation, RDF, 3D MoRSE and GETAWAY parameters are the useful parameters for modeling the EGFR inhibitory activity for the present set of compounds.

2. MATS1e and MATS3p plays a dominating role for prediction of antitumor activity.

\section{Acknowledgement}

The corresponding author is thankful to UGC. This work was financially supported by the UGC MRP.

\section{References}

[1]. C.H. Yarbro, M. Goodman, M.H. Frogge. Cancer Nursing Principles and Practice (6th edition); Boston, Jones \& Bartlett Publishers. (2005), 1879, ISBN 0815169906.

[2]. J. Ferlay, H.R. Shin, F. Bray, D. Forman, C. Mathers, D.M. Parkin ,GLOBOCAN 2008 v1.2. Cancer Incidence and Mortality Worldwide: IARC CancerBase No. 10. Lyon, France: International Agency for Research on Cancer, Available from: http://globocan. iarc.fr, accessed on day/month/year. (2010).

[3]. M.E. Bracke, R.M.L. Van Cauwenberge, M.M. Mareel, Clin. Metastasis, 2, (1984), 161

[4]. R. Giavazzi, A. Garofalo, G. Damia, S. Garattini, M. D’Incalci, British J. of Cancer, 57(3), (1988), 277.

[5]. S. Mukherjee, V. Kumar, A.K. Prasad, H.G. Raj, M.E. Bracke, C.E. Olsen, S.C. Jain, V.S. Parmar, Bioorg. Med. Chem., 9(2), (2001), 337.

[6]. B.W. Vanhoecke, H.T. Depypere, A. De Beyter, S.K. Sharma, V.S. Parmar, D. De Keukeleire, M.E. Bracke, Pure App. Chem. , 71(1), (2005), 65 .

[7]. L. Sartor, E. Pezzato, I. Dell'Aica, R. Caniato, S. Biggin, S. Garbisa, Biochem. Pharm. 64(2), (2002), 229.

[8]. D.S. Salomon, R. Brandt, F. Ciardiello, Crit. Rev. Oncol. Hematol. 19, (1995), 183-232.

[9]. G.W. Rewcastle, W.A. Denny, A.J. Bridges, H.R. Zhou, D.R. Cody, A. Mcmichael, D.W. Fry, J. Med. Chem., 38, (1995), 34823487

[10]. A.M. Thompson, A.J. Bridges, D.W. Fry, A.J. Kraker, W.A. Denny, J. Med. Chem., 38, (1995), $3780-3788$.

[11]. J.B. Smaill, B.D. Palmer, G.W. Rewcastle, W.A. Denny, D.J. McNamara, E.M. Dobrusin, A.J. Bridges, H.R. Zhou, H.D.H. Showalter, R.T. Winters, W.R. Leopold, D.W. Fry, J.M. Nelson, V. Slintak, W.L. Elliot, B.J. Roberts, P.W. Vincent, S.J. Patmore, J. Med. Chem. 42, (1999), 1803-1815.

[12]. G.W. Rewcastle, B.D. Palmer, A.J. Bridges, H.D.H. Showalter, S. Li, J. Nelson, A. McMichael, A.J., Kraker, D.W. Fry, W.A. Denny, J. Med. Chem., 39, (1996), 918-928.

[13]. J.B. Smaill, H.D.H. Showalter, H.R. Zhou, A.J. Bridges, D.J. McNamara, D.W. Fry, J.M. Nelson, V. Sherwood, P.W. Vincent, B.J. Roberts, W.L. Elliott, W.A. Denny, J. Med. Chem. 44,(2001) 429-440.

[14]. H. Assefa, S. Kamath, J.K. Buolamwini, J. Comp. Aided Mol. Des., 17, (2003), 475-493.

[15]. D.V. Pednekar, M.A. Kelkar, S.R. Pimple, K.G. Akamanchi, Med. Chem. Res., 13, (2004), 605-618.

[16]. J. Stamos, M.X. Sliwkowski, C. Eigenbrot. J. Biol. Chem., 277 ,(2002), 46265-46272.

[17]. F.A. Pasha, M. Muddassar, A.K. Srivastava, S.J. Cho, J.Mol. Model. 16, (2010), 253-277.

[18]. DRAGON software for calculation of topological indices, www.disat.unimib.it

[19]. NCSS statistical analysis software, www.ncss.com

[20]. L. Pogliani, AminoAcids, 6, (1994), 141.

[21]. L. Pogliani, J. Phys. Chem., 100, (1996), 18065.

[22]. L. Pogliani, Chem. Rev., 100, (2000), 3827. 
<smiles>[X]c1ccc(Nc2ncnc3ccccc23)cc1[X]</smiles>

Fig.1 Basic structure of anilinoquinolines.

Table .1 Structural details and $\mathrm{pIC}_{50}$ values for the compounds used in present study.

\begin{tabular}{|c|c|c|c|}
\hline Compound no. & $\mathbf{X}$ & $\mathbf{Y}$ & $\mathrm{pIC}_{50}$ \\
\hline 1 & $\mathrm{H}$ & $\mathrm{H}$ & 6.46 \\
\hline 2 & $3-\mathrm{F}$ & $\mathrm{H}$ & 7.25 \\
\hline 3 & $3-\mathrm{Cl}$ & $\mathrm{H}$ & 7.64 \\
\hline 4 & $3-\mathrm{Br}$ & $\mathrm{H}$ & 7.57 \\
\hline 5 & $3-I$ & $\mathrm{H}$ & 7.1 \\
\hline 6 & $3-\mathrm{CF}_{3}$ & $\mathrm{H}$ & 6.24 \\
\hline 7 & $\mathrm{H}$ & 6-OMe & 7.26 \\
\hline 8 & $3-\mathrm{Br}$ & 6-OMe & 7.52 \\
\hline 9 & $\mathrm{H}$ & 6- $\mathrm{NH}_{2}$ & 6.11 \\
\hline 10 & $3-\mathrm{CF}_{3}$ & $6-\mathrm{NH}_{2}$ & 6.24 \\
\hline 11 & $3-\mathrm{Br}$ & $6-\mathrm{NH}_{2}$ & 9.11 \\
\hline 12 & $\mathrm{H}$ & $6-\mathrm{NO}_{2}$ & 5.3 \\
\hline 13 & $3-\mathrm{Br}$ & $6-\mathrm{NO}_{2}$ & 6.05 \\
\hline 14 & $\mathrm{H}$ & 7-OMe & 6.92 \\
\hline 15 & $3-\mathrm{Br}$ & 7-OMe & 8 \\
\hline 16 & $\mathrm{H}$ & 7- $\mathrm{NH}_{2}$ & 7 \\
\hline 17 & $3-\mathrm{F}$ & $7-\mathrm{NH}_{2}$ & 8.7 \\
\hline 18 & $3-\mathrm{Cl}$ & 7- $\mathrm{NH}_{2}$ & 9.6 \\
\hline 19 & $3-\mathrm{Br}$ & 7- $\mathrm{NH}_{2}$ & 10 \\
\hline 20 & 3 -I & 7- $\mathrm{NH}_{2}$ & 9.46 \\
\hline 21 & $3-\mathrm{CF}_{3}$ & 7- $\mathrm{NH}_{2}$ & 8.48 \\
\hline 22 & $\mathrm{H}$ & $7-\mathrm{NO}_{2}$ & 4.92 \\
\hline 23 & $3-\mathrm{F}$ & $7-\mathrm{NO}_{2}$ & 5.22 \\
\hline 24 & $3-\mathrm{Cl}$ & $7-\mathrm{NO}_{2}$ & 6.09 \\
\hline 25 & $3-\mathrm{Br}$ & $7-\mathrm{NO}_{2}$ & 6 \\
\hline 26 & $3-I$ & $7-\mathrm{NO}_{2}$ & 6.27 \\
\hline 27 & $\mathrm{H}$ & 6,7-di-OMe & 7.54 \\
\hline 28 & $3-\mathrm{F}$ & 6,7-di-OMe & 8.42 \\
\hline 29 & $3-\mathrm{Cl}$ & 6,7-di-OMe & 9.51 \\
\hline 30 & $3-\mathrm{Br}$ & 6,7-di-OMe & 10.6 \\
\hline 31 & $3-I$ & 6,7-di-OMe & 9.05 \\
\hline 32 & $3-\mathrm{CF}_{3}$ & 6,7-di-OMe & 9.62 \\
\hline 33 & $3-\mathrm{Br}$ & 6-NHMe & 8.4 \\
\hline 34 & $3-\mathrm{Br}$ & 6- $\mathrm{NMe}_{2}$ & 7.08 \\
\hline 35 & $3-\mathrm{Br}$ & $7-\mathrm{OH}$ & 8.33 \\
\hline 36 & $3-\mathrm{Br}$ & 7-NHMe & 8.16 \\
\hline 37 & $3-\mathrm{Br}$ & 7-NHC2 $\mathrm{H}_{5}$ & 7.92 \\
\hline 38 & $3-\mathrm{Br}$ & $7-\mathrm{NMe}_{2}$ & 7.96 \\
\hline 39 & $3-\mathrm{Br}$ & 6,7-di- $\mathrm{NH}_{2}$ & 9.92 \\
\hline 40 & $3-\mathrm{Br}$ & $6-\mathrm{NH}_{2}, 7-\mathrm{NHMe}$ & 9.16 \\
\hline 41 & $3-\mathrm{Br}$ & $6-\mathrm{NH}_{2}, 7-\mathrm{NMe}_{2}$ & 6.8 \\
\hline 42 & $3-\mathrm{Br}$ & 6- $\mathrm{NH}_{2}, 7-\mathrm{OMe}$ & 8.42 \\
\hline 43 & $3-\mathrm{Br}$ & 6- $\mathrm{NH}_{2}, 7-\mathrm{Cl}$ & 8.19 \\
\hline 44 & $3-\mathrm{Br}$ & $6-\mathrm{NO}_{2}, 7-\mathrm{NH}_{2}$ & 7.28 \\
\hline 45 & $3-\mathrm{Br}$ & $6-\mathrm{NO}_{2}, 7-\mathrm{NHMe}$ & 7.17 \\
\hline 46 & $3-\mathrm{Br}$ & $6-\mathrm{NO}_{2}, 7-\mathrm{NMe}_{2}$ & 5.7 \\
\hline 47 & $3-\mathrm{Br}$ & $6-\mathrm{NO}_{2}, 7-\mathrm{OMe}$ & 7.82 \\
\hline 48 & $3-\mathrm{Br}$ & $6-\mathrm{NO}_{2}, 7-\mathrm{Cl}$ & 7.6 \\
\hline 49 & $3-\mathrm{Br}$ & 6,7-di-OHa & 9.77 \\
\hline 50 & $3-\mathrm{Br}$ & 6,7-di- $\mathrm{OC}_{2} \mathrm{H}_{5}$ & 11.22 \\
\hline 51 & $3-\mathrm{Br}$ & $6,7-\mathrm{di}-\mathrm{OC}_{3} \mathrm{H}_{7}$ & 9.77 \\
\hline 52 & $3-\mathrm{Br}$ & $6,7-\mathrm{di}-\mathrm{OC}_{4} \mathrm{H}_{9}$ & 6.98 \\
\hline 53 & $3-\mathrm{Br}$ & 5,6-di-OMe & 5.86 \\
\hline
\end{tabular}


QSAR studies of some anilinoquinolines for their antitumor activity as EGFR inhibitors

\begin{tabular}{|c|c|c|c|}
\hline 54 & $3-\mathrm{Br}$ & $5,6,7-$ tri-OMe & 9.17 \\
\hline 55 & $2-\mathrm{Br}$ & $6,7-$ di-OMe & 6.89 \\
\hline 56 & $4-\mathrm{Br}$ & $6,7-$ di-OMe & 9.02 \\
\hline 57 & $3,4-\mathrm{di}-\mathrm{Br}$ & $6,7-$ di-OMe & 10.14 \\
\hline 58 & $3,5-\mathrm{di}-\mathrm{Br}$ & $6,7-$ di-OMe & 6.95 \\
\hline
\end{tabular}

Table 2. Calculated values of 2D autocorrelation, RDF, 3D MoRSE, WHIM, and GETAWAY descriptors for the compounds used in present study.

\begin{tabular}{|c|c|c|c|c|c|c|c|}
\hline S.No. & MATS1e & MATS3p & RDF110u & RDF095s & Mor29u & G3m & H1i \\
\hline 1 & $\begin{array}{l}-0.046 \\
\end{array}$ & -0.122 & 0.000 & 0.097 & 0.176 & 0.295 & 2.613 \\
\hline 2 & -0.051 & -0.117 & 0.000 & 0.050 & 0.266 & 0.193 & 2.652 \\
\hline 3 & -0.067 & -0.152 & 0.000 & 12.376 & 0.164 & 0.233 & 2.585 \\
\hline 4 & -0.073 & -0.141 & 0.001 & 6.497 & 0.263 & 0.233 & 2.562 \\
\hline 5 & -0.061 & -0.099 & 0.587 & 5.760 & 0.199 & 0.255 & 2.533 \\
\hline 6 & -0.044 & -0.214 & 0.661 & 37.934 & 0.191 & 0.220 & 2.188 \\
\hline 7 & -0.045 & -0.119 & 0.000 & 0.227 & 0.150 & 0.200 & 2.359 \\
\hline 8 & $\begin{array}{l}-0.066 \\
\end{array}$ & -0.142 & 0.001 & 6.151 & 0.229 & 0.200 & 2.536 \\
\hline 9 & $\begin{array}{l}-0.171 \\
\end{array}$ & -0.184 & 0.000 & 0.334 & 0.082 & 0.307 & 2.765 \\
\hline 10 & -0.086 & -0.256 & 0.849 & 35.521 & 0.000 & 0.283 & 2.276 \\
\hline 11 & -0.186 & -0.189 & 0.002 & 6.914 & 0.101 & 0.188 & 2.714 \\
\hline 12 & 0.207 & -0.178 & 0.000 & 0.826 & 0.076 & 0.224 & 2.750 \\
\hline 13 & 0.182 & -0.186 & 0.000 & 5.764 & 0.084 & 0.206 & 2.695 \\
\hline 14 & -0.045 & -0.119 & 0.005 & 2.100 & 0.245 & 0.200 & 2.579 \\
\hline 15 & -0.066 & -0.142 & 0.000 & 9.067 & 0.371 & 0.184 & 2.580 \\
\hline 16 & -0.171 & -0.184 & 0.000 & 1.043 & 0.152 & 0.245 & 2.749 \\
\hline 17 & -0.116 & -0.179 & 0.109 & 1.779 & 0.094 & 0.197 & 2.785 \\
\hline 18 & -0.164 & -0.211 & 0.018 & 12.174 & 0.030 & 0.206 & 2.717 \\
\hline 19 & -0.186 & -0.189 & 0.000 & 7.948 & 0.088 & 0.169 & 2.695 \\
\hline 20 & -0.190 & -0.121 & 0.429 & 7.269 & 0.069 & 0.188 & 2.666 \\
\hline 21 & -0.086 & -0.256 & 1.790 & 25.427 & 0.148 & 0.197 & 2.820 \\
\hline 22 & 0.207 & -0.178 & 0.939 & 3.947 & 0.234 & 0.206 & 2.746 \\
\hline 23 & 0.111 & -0.172 & 0.739 & 0.936 & 0.253 & 0.197 & 2.790 \\
\hline 24 & 0.159 & -0.208 & 0.000 & 3.608 & 0.246 & 0.188 & 2.728 \\
\hline 25 & 0.182 & -0.186 & 0.000 & 9.482 & 0.336 & 0.197 & 2.704 \\
\hline 26 & 0.206 & -0.120 & 0.004 & 4.865 & 0.319 & 0.188 & 2.683 \\
\hline 27 & -0.052 & -0.105 & 0.000 & 2.613 & 0.326 & 0.246 & 2.546 \\
\hline 28 & -0.065 & -0.102 & 0.025 & 1.753 & 0.447 & 0.191 & 2.614 \\
\hline 29 & -0.071 & -0.136 & 0.003 & 11.858 & 0.460 & 0.177 & 2.666 \\
\hline 30 & -0.070 & -0.132 & 0.470 & 15.889 & 0.330 & 0.177 & 2.668 \\
\hline 31 & -0.059 & -0.101 & 0.041 & 6.550 & 0.543 & 0.162 & 2.622 \\
\hline 32 & -0.066 & -0.171 & 2.643 & 73.531 & 0.522 & 0.173 & 2.665 \\
\hline 33 & -0.109 & -0.109 & 0.000 & 8.839 & 0.226 & 0.214 & 2.559 \\
\hline 34 & -0.040 & -0.195 & 0.005 & 16.806 & 0.038 & 0.198 & 2.382 \\
\hline 35 & -0.160 & -0.163 & 0.000 & 8.544 & 0.158 & 0.209 & 2.628 \\
\hline 36 & -0.109 & -0.109 & 0.000 & 16.058 & 0.369 & 0.222 & 2.600 \\
\hline 37 & -0.088 & -0.004 & 2.740 & 16.360 & 0.082 & 0.184 & 2.519 \\
\hline 38 & -0.040 & -0.195 & 0.008 & 16.883 & 0.564 & 0.177 & 2.535 \\
\hline 39 & -0.276 & -0.219 & 0.000 & 10.549 & 0.227 & 0.184 & 2.786 \\
\hline 40 & -0.199 & -0.140 & 0.003 & 11.186 & 0.218 & 0.193 & 2.650 \\
\hline 41 & -0.132 & -0.216 & 0.001 & 16.226 & 0.384 & 0.214 & 2.576 \\
\hline 42 & -0.143 & -0.177 & 0.001 & 11.535 & 0.216 & 0.164 & 2.651 \\
\hline 43 & -0.178 & -0.205 & 0.000 & 7.094 & 0.129 & 0.206 & 2.692 \\
\hline 44 & 0.094 & -0.219 & 0.012 & 11.881 & 0.272 & 0.184 & 2.770 \\
\hline
\end{tabular}


QSAR studies of some anilinoquinolines for their antitumor activity as EGFR inhibitors

\begin{tabular}{|l|l|l|l|l|l|l|l|}
\hline 45 & 0.131 & -0.137 & 0.001 & 11.248 & 0.050 & 0.179 & 2.634 \\
\hline 46 & 0.163 & -0.215 & 0.001 & 15.532 & 0.250 & 0.187 & 2.557 \\
\hline 47 & 0.113 & -0.175 & 0.064 & 12.855 & 0.166 & 0.180 & 2.645 \\
\hline 48 & 0.141 & -0.204 & 0.000 & 8.518 & 0.049 & 0.169 & 2.672 \\
\hline 49 & -0.216 & -0.182 & 0.000 & 8.698 & 0.173 & 0.197 & 2.676 \\
\hline 50 & -0.043 & 0.128 & 1.364 & 18.212 & 0.090 & 0.169 & 2.543 \\
\hline 51 & -0.024 & 0.068 & 4.719 & 29.548 & -0.171 & 0.177 & 2.548 \\
\hline 52 & -0.010 & 0.071 & 8.684 & 32.772 & -0.415 & 0.182 & 2.524 \\
\hline 53 & -0.070 & -0.126 & 1.904 & 13.297 & 0.605 & 0.205 & 2.709 \\
\hline 54 & -0.075 & -0.112 & 0.346 & 12.074 & 0.378 & 0.165 & 2.775 \\
\hline 55 & -0.070 & -0.090 & 1.679 & 4.607 & 0.273 & 0.191 & 2.405 \\
\hline 56 & -0.070 & -0.132 & 0.285 & 13.644 & 0.392 & 0.184 & 2.733 \\
\hline 57 & -0.086 & 0.020 & 0.828 & 23.506 & 0.452 & 0.177 & 2.639 \\
\hline 58 & -0.086 & -0.174 & 1.367 & 16.717 & 0.499 & 0.198 & 2.723 \\
\hline
\end{tabular}

Table 3. Correlation matrix

\begin{tabular}{|c|c|c|c|c|c|c|c|c|}
\hline & pIC50 & MATS1e & MATS3p & RDF110u & RDF095s & Mor29u & G3m & H1i \\
\hline pIC50 & 1.000 & & & & & & & \\
\hline MATS1e & -0.602 & 1.000 & & & & & & \\
\hline MATS3p & 0.331 & -0.030 & 1.000 & & & & & \\
\hline RDF110u & 0.035 & 0.022 & 0.571 & 1.000 & & & & \\
\hline RDF095s & 0.244 & -0.079 & 0.095 & 0.521 & 1.000 & & & \\
\hline Mor29u & 0.041 & -0.019 & -0.176 & -0.422 & -0.002 & 1.000 & & \\
\hline G3m & -0.462 & -0.122 & -0.218 & -0.163 & -0.183 & -0.163 & 1.000 & \\
\hline H1i & 0.124 & 0.048 & -0.208 & -0.160 & -0.291 & 0.164 & -0.181 & 1.000 \\
\hline
\end{tabular}

Table 4. Regression parameters and quality of correlations

\begin{tabular}{|c|c|c|c|c|c|c|c|c|}
\hline $\begin{array}{c}\text { Model } \\
\text { No. }\end{array}$ & $\begin{array}{c}\text { Parameters } \\
\text { Used }\end{array}$ & $\mathrm{A}_{\mathrm{i}}=(1 \ldots \ldots 6)$ & B & $\mathrm{Se}$ & $\mathrm{R}^{2}$ & $\mathrm{R}_{\mathrm{A}}^{2}$ & $\mathrm{~F}$ & $\mathrm{Q}=\mathrm{R} / \mathrm{Se}$ \\
\hline 1 & MATS1e & $-7.4635( \pm 1.3218)$ & 7.4706 & 0.1518 & 0.3628 & 0.3514 & 31.883 & 3.968 \\
\hline 2 & $\begin{array}{c}\text { G3m } \\
\text { MATS1e }\end{array}$ & $\begin{array}{c}-26.3556( \pm 3.8767) \\
-8.2836( \pm 0.9905\end{array}$ & 12.7199 & 0.1129 & 0.6538 & 0.6412 & 51.925 & 7.162 \\
\hline 3 & $\begin{array}{c}\text { G3m } \\
\text { MATS1e } \\
\text { MATS3p }\end{array}$ & $\begin{array}{c}-24.1464( \pm 3.7812) \\
-8.1400( \pm 0.9433) \\
3.9631( \pm 1.5146)\end{array}$ & 12.8520 & 0.1074 & 0.6927 & 0.6756 & 40.579 & 7.749 \\
\hline 4 & $\begin{array}{c}\text { G3m } \\
\text { MATS1e } \\
\text { MATS3p } \\
\text { RDF110u }\end{array}$ & $\begin{array}{c}-24.5216( \pm 3.6152) \\
-8.0460( \pm 0.9019) \\
6.3734( \pm 1.7418) \\
-0.2308( \pm 0.0929)\end{array}$ & 13.4100 & 0.1025 & 0.7248 & 0.7040 & 34.895 & 8.306 \\
\hline 5 & $\begin{array}{l}\text { G3m } \\
\text { MATS1e } \\
\text { MATS3p } \\
\text { RDF095s } \\
\text { RDF110u }\end{array}$ & $\begin{array}{c}-22.2852( \pm 3.3394) \\
-7.6033( \pm 0.8275) \\
8.2819( \pm 1.6698) \\
0.0356( \pm 0.0101) \\
-0.4418( \pm 0.1033)\end{array}$ & 12.9503 & 0.0930 & 0.7779 & 0.7566 & 36.429 & 9.484 \\
\hline 6 & $\begin{array}{c}\text { G3m } \\
\text { H1i } \\
\text { MATS1e } \\
\text { MATS3p } \\
\text { RDF095s } \\
\text { RDF110u }\end{array}$ & $\begin{array}{c}-18.9191( \pm 3.2170) \\
2.6503( \pm 0.7941) \\
-7.4943( \pm 0.7577) \\
9.8815( \pm 1.6009) \\
0.0477( \pm 0.0099) \\
-0.4949( \pm 0.0958)\end{array}$ & 5.4334 & 0.0851 & 0.8177 & 0.7963 & 38.134 & 10.626 \\
\hline 7 & $\begin{array}{c}\text { G3m } \\
\text { H1i } \\
\text { MATS1e } \\
\text { MATS3p } \\
\text { Mor29u } \\
\text { RDF095s } \\
\text { RDF110u }\end{array}$ & $\begin{array}{c}-20.5758( \pm 2.8191) \\
3.2238( \pm 0.7024) \\
-7.4577( \pm 0.6576) \\
11.0537( \pm 1.4168) \\
-2.1918( \pm 0.5205) \\
0.0607( \pm 0.0091) \\
-0.7058( \pm 0.0971)\end{array}$ & 4.8803 & 0.0738 & 0.8654 & 0.8466 & 45.943 & 12.605 \\
\hline
\end{tabular}


Table 5. Observed and Estimated $\mathrm{pIC}_{50}$ values using model no 7.(Table 4).

\begin{tabular}{|c|c|c|c|}
\hline Comp. No. & Obs. pIC ${ }_{50}$ & Est. pIC ${ }_{50}$ & Residual \\
\hline 1 & 6.46 & 5.85 & 0.61 \\
\hline 2 & 7.25 & 7.97 & -0.72 \\
\hline 3 & 7.64 & 7.63 & 0.01 \\
\hline 4 & 7.57 & 7.15 & 0.42 \\
\hline 5 & 7.10 & 6.66 & 0.44 \\
\hline 6 & 6.24 & 6.79 & -0.55 \\
\hline 7 & 7.26 & 7.08 & 0.19 \\
\hline 8 & 7.52 & 7.73 & -0.21 \\
\hline 9 & 6.11 & 6.56 & -0.45 \\
\hline 10 & 6.24 & 5.76 & 0.48 \\
\hline 11 & 9.11 & 9.26 & -0.15 \\
\hline 12 & 5.30 & 5.51 & -0.21 \\
\hline 13 & 6.05 & 6.08 & -0.03 \\
\hline 14 & 6.92 & 7.69 & -0.77 \\
\hline 15 & 8.00 & 8.07 & -0.07 \\
\hline 16 & 7.00 & 7.67 & -0.67 \\
\hline 17 & 8.70 & 8.52 & 0.18 \\
\hline 18 & 9.60 & 8.95 & 0.65 \\
\hline 19 & 10.00 & 9.68 & 0.32 \\
\hline 20 & 9.46 & 9.67 & -0.21 \\
\hline 21 & 8.48 & 7.69 & 0.80 \\
\hline 22 & 4.92 & 5.05 & -0.13 \\
\hline 23 & 5.22 & 6.07 & -0.85 \\
\hline 24 & 6.09 & 6.00 & 0.09 \\
\hline 25 & 6.00 & 5.97 & 0.03 \\
\hline 26 & 6.27 & 6.39 & -0.12 \\
\hline 27 & 7.54 & 6.70 & 0.84 \\
\hline 28 & 8.42 & 7.84 & 0.58 \\
\hline 29 & 9.51 & 8.57 & 0.94 \\
\hline 30 & 10.60 & 8.81 & 1.79 \\
\hline 31 & 9.05 & 8.50 & 0.55 \\
\hline 32 & 9.62 & 9.97 & -0.35 \\
\hline 33 & 8.40 & 8.38 & 0.02 \\
\hline 34 & 7.08 & 7.56 & -0.48 \\
\hline 35 & 8.33 & 8.62 & -0.29 \\
\hline 36 & 8.16 & 8.47 & -0.31 \\
\hline 37 & 7.92 & 8.71 & -0.79 \\
\hline 38 & 7.96 & 7.34 & 0.62 \\
\hline 39 & 9.92 & 9.86 & 0.06 \\
\hline 40 & 9.16 & 9.59 & -0.43 \\
\hline 41 & 6.80 & 7.52 & -0.72 \\
\hline 42 & 8.42 & 9.39 & -0.97 \\
\hline 43 & 8.19 & 8.53 & -0.34 \\
\hline 44 & 7.28 & 7.02 & 0.26 \\
\hline 45 & 7.17 & 7.77 & -0.60 \\
\hline 46 & 5.70 & 6.08 & -0.38 \\
\hline 47 & 7.82 & 7.30 & 0.52 \\
\hline 48 & 7.60 & 7.12 & 0.48 \\
\hline 49 & 9.77 & 9.20 & 0.57 \\
\hline 50 & 11.22 & 11.28 & -0.06 \\
\hline 51 & 9.77 & 9.22 & 0.55 \\
\hline 52 & 6.98 & 6.90 & 0.08 \\
\hline 53 & 5.86 & 6.66 & -0.80 \\
\hline 54 & 9.17 & 9.41 & -0.24 \\
\hline 55 & 6.89 & 6.73 & 0.16 \\
\hline 56 & 9.02 & 8.74 & 0.28 \\
\hline 57 & 10.14 & 10.46 & -0.32 \\
\hline 58 & 6.95 & 7.26 & -0.31 \\
\hline
\end{tabular}

Table 6. Cross validated parameters for the best obtained models.

\begin{tabular}{|c|c|c|c|c|c|}
\hline Model No. & Parameters used & PRESS/SSY & $\mathrm{R}_{\mathrm{cv}}^{2}$ & S $_{\text {PRESS }}$ & PSE \\
\hline 1 & MATS1e & 1.7564 & -0.7564 & 1.1855 & 0.1649 \\
\hline 2 & $\begin{array}{c}\text { G3m } \\
\text { MATS1e }\end{array}$ & 0.5296 & 0.4704 & 0.8818 & 0.8587 \\
\hline 3 & $\begin{array}{c}\text { G3m } \\
\text { MATS1e }\end{array}$ & 0.4436 & 0.5564 & 0.7028 & 0.8089 \\
\hline
\end{tabular}


QSAR studies of some anilinoquinolines for their antitumor activity as EGFR inhibitors

\begin{tabular}{|c|c|c|c|c|c|}
\hline & MATS3p & & & & \\
\hline 4 & $\begin{array}{c}\text { G3m } \\
\text { MATS1e } \\
\text { MATS3p } \\
\text { RDF110u }\end{array}$ & 0.3797 & 0.6203 & 0.8008 & 0.7655 \\
\hline 5 & $\begin{array}{c}\text { G3m } \\
\text { MATS1e } \\
\text { MATS3p } \\
\text { RDF095s } \\
\text { RDF110u }\end{array}$ & 0.2855 & 0.7145 & 0.7263 & 0.6877 \\
\hline 6 & $\begin{array}{c}\text { G3m } \\
\text { H1i } \\
\text { MATS1e } \\
\text { MATS3p } \\
\text { RDF095s } \\
\text { RDF110u }\end{array}$ & 0.2229 & 0.7771 & 0.6644 & 0.6230 \\
\hline 7 & $\begin{array}{c}\text { G3m } \\
\text { H1i } \\
\text { MATS1e } \\
\text { MATS3p } \\
\text { Mor29u } \\
\text { RDF095s } \\
\text { RDF110u }\end{array}$ & 0.1555 & 0.8445 & 0.5765 & 0.5353 \\
\hline
\end{tabular}

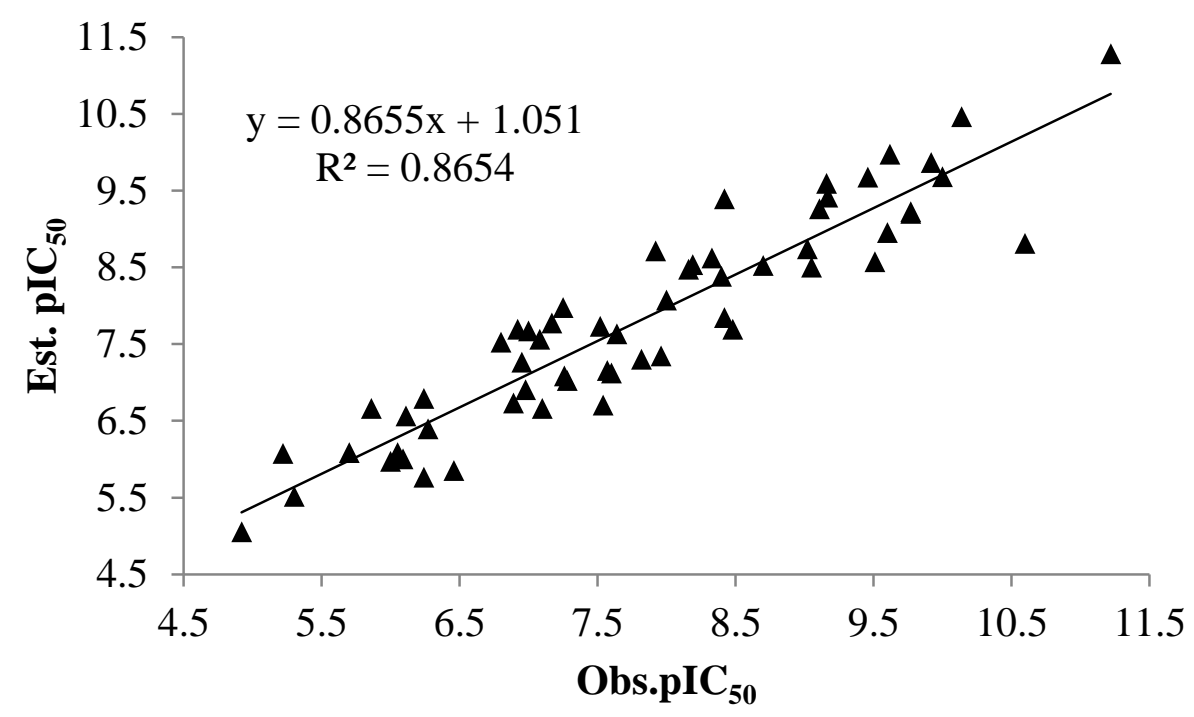

Figure 2. Correlation between Observed and estimated $\mathrm{pIC}_{50}$ activity using model 7(Table 4).

Table 7. Ridge analysis for the best seven-parametric model.

\begin{tabular}{|c|c|c|c|c|c|}
\hline $\begin{array}{c}\text { Model } \\
\text { No. }\end{array}$ & Parameters used & VIF & Tolerence & Eigenvalue & Condition no. \\
\hline 7 & MATS1e & 1.0464 & 0.9556 & 2.1416 & 1.00 \\
& MATS3p & 1.9491 & 0.5131 & 1.3253 & 1.62 \\
& RDF110u & 3.1378 & 0.3187 & 0.9363 & 1.94 \\
& RDF095s & 2.0512 & 0.4875 & 0.7875 & 2.29 \\
& Mor29u & 1.4864 & 0.6728 & 0.5377 & 2.72 \\
& G3m & 1.2557 & 0.7964 & 0.1685 & 3.98 \\
& H1i & 1.3308 & 0.7515 & 12.70 \\
\hline
\end{tabular}




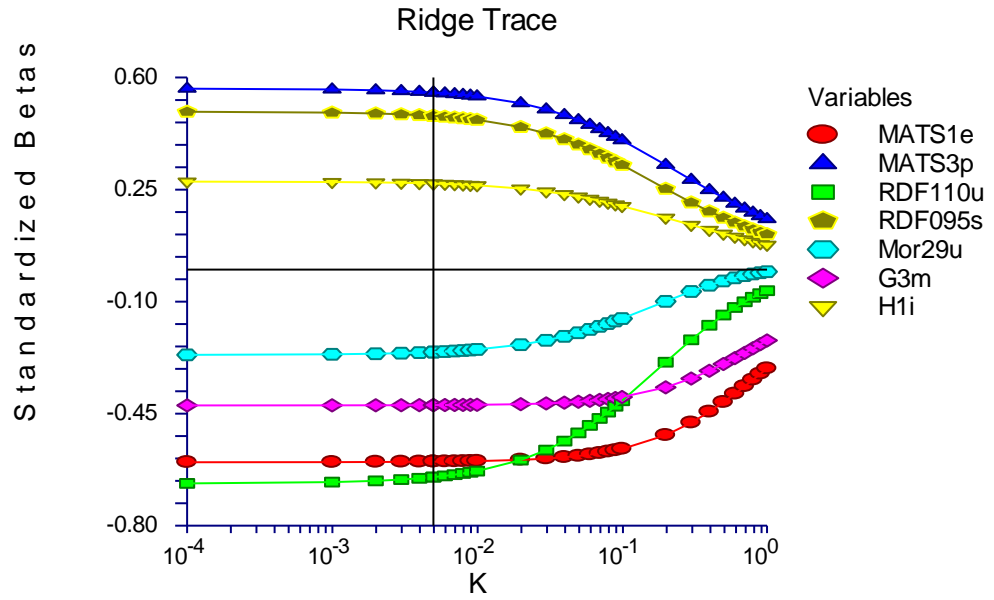

Fig 3: Ridge trace for seven variable model 7 (Table 4)

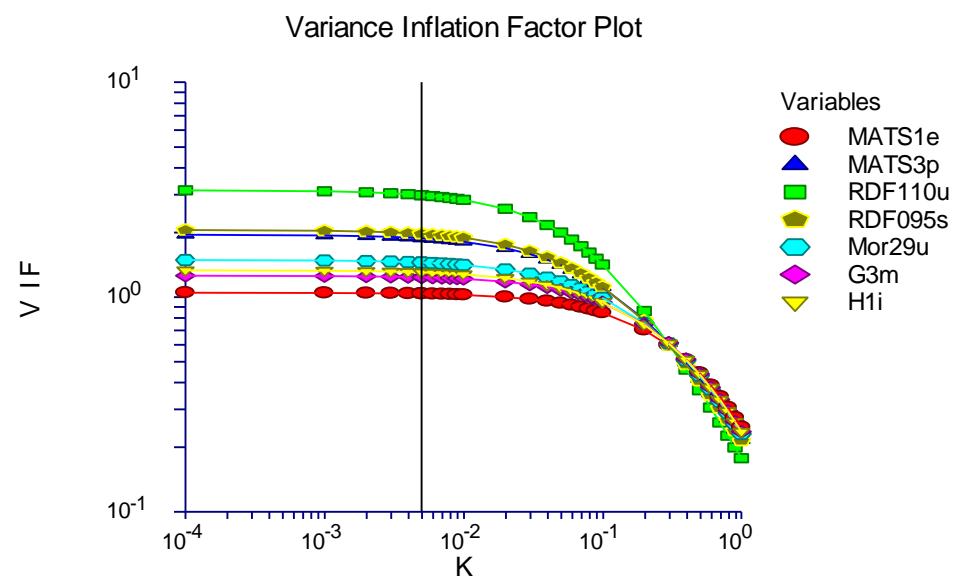

Fig 4:VIF plot for seven variable model-7(Table 4) 